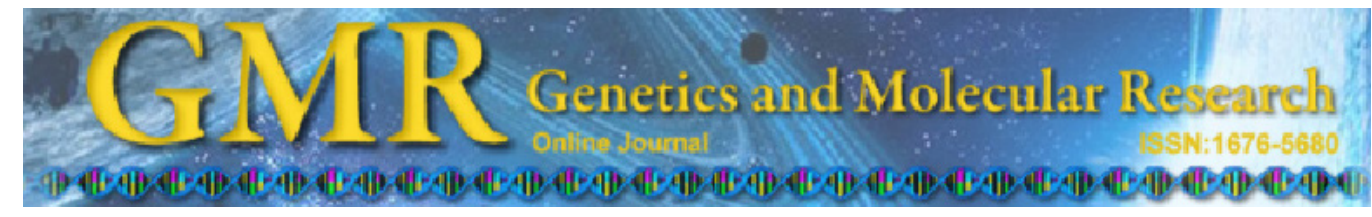

\title{
Molecular characterization and expression analysis of the Lrh-1 gene in Chinese Hu sheep
}

\author{
L.H. Wang ${ }^{1,2}$, W. Zhang ${ }^{2}$, J.L. Ji ${ }^{2}$, Q.X. Gao ${ }^{2}$, S.H. Xiao ${ }^{1}$ and F. Wang ${ }^{1}$ \\ ${ }^{1}$ Center of Embryo Engineering and Technology, \\ College of Animal Science and Technology, \\ Nanjing Agricultural University, Nanjing, Jiangsu, China \\ ${ }^{2}$ Jiangsu Animal Husbandry and Veterinary College, Taizhou, Jiangsu, China \\ Corresponding author: F. Wang \\ E-mail: caeet@njau.edu.cn
}

Genet. Mol. Res. 12 (2): 1490-1500 (2013)

Received July 31, 2012

Accepted January 17, 2013

Published May 6, 2013

DOI http://dx.doi.org/10.4238/2013.May.6.2

\begin{abstract}
Liver receptor homologue 1 (Lrh-1) is a member of the nuclear receptor belonging to the second subfamily of the nuclear receptor family 5A (NR5A), also named NR5A2, which is important for lipid homeostasis, embryogenesis, and regulation of aromatics. The present study aimed to understand the sequence of ovine Lrh-1 and the expression traits in reproductive organ tissues. Initially, we cloned Lrh-1 from the liver of $\mathrm{Hu}$ sheep through degenerate primer of reverse transcriptionpolymerase chain reaction and rapid amplification of cDNA ends. Characteristic functional domains of DNA binding and ligand binding, conserved among transcription factors of the nuclear receptor superfamily, were identified in Lrh-1 of Hu sheep. The Lrh-1 protein levels in the tissues detected by Western blotting correlated significantly with the transcript levels measured by quantitative real-time polymerase chain reaction (qRTPCR). To understand the Lrh-1 expression change in the hypothalamus and hypophysis during the estrous cycle, we analyzed the expression pattern of Lrh-1 mRNA and protein by qRT-PCR and Western blotting, respectively. This analysis revealed that Lrh-1 expression in the hypothalamus was highest during the metestrus phase, while the Lrh-1 level was similar during other phases. In the hypophysis, the expression was significantly different during the 4 phases of the estrous cycle but highest during the estrus phase,
\end{abstract}


significantly correlating with FSH concentration. These results indicate that Lrh-1 expression is correlated with gonadotropic hormone secretion, influencing follicular formation in the ovary.

Key words: Lrh-1; Hu sheep; qRT-PCR; Western blot

\section{INTRODUCTION}

Liver receptor homolog-1 (Lrh-1) is an orphan nuclear receptor of the NR5A subfamily of nuclear receptors (Becker-Andre et al., 1993; Galarneau et al., 1996; Li et al., 1998; Nitta et al., 1999), which includes 2 major subgroups of related genes with separate functions and expression patterns in higher vertebrates. The NR5A2 subgroup contains Lrh-1, or $\alpha$-fetoprotein transcription factor, which regulates the expression of the $\alpha$-fetoprotein (Galarneau et al., 1996) and is involved in cholesterol metabolism in mammals (Nitta et al., 1999).

Lrh-1 plays a central role in lipid homeostasis, embryogenesis, regulation of aromatases (Fayard et al., 2004; Sridevi et al., 2011), and regulation of the 1st and rate-limiting step in the classical or neutral pathway of bile acid biosynthesis by transcriptional activation of cholesterol-7alpha-hydroxylase, CYP7A1 (Nitta et al., 1999; Goodwin et al., 2000; Lu et al., 2000). In addition, Lrh-1 is implicated in the regulation of key transcription factors involved in hepatic and pancreatic development, such as the hepatic nuclear factors (HNF)3 beta, HNF-4alpha, and HNF-1alpha (Pare et al., 2001). Lrh-1 regulates the expression of aromatase (CYP19) in ovarian and adipose tissues (Clyne et al., 2002) and adiponectin in adipocytes (Iwaki et al., 2003). Moreover, Lrh-1 regulates the expression of apolipoprotein AI (Delerive et al., 2004), cholesteryl ester transfer protein (Luo et al., 2001), and scavenger receptor class B type I (Schoonjans et al., 2002), thereby implicating this receptor in highdensity lipoprotein remodeling and cholesterol transport (Higashiyama et al., 2007; Sridevi et al., 2011). However, to the best of our knowledge, there are no reports on the ovine Lrh-1 gene. This prompted us to characterize Lrh-1 in Hu sheep, an ovine species widely raised in the Taihu Lake area of China that covers the provinces of Jiangsu and Zhejiang and the vicinity of Shanghai. This sheep breed is known for its beautiful lambskin, early sexual maturity, and high fecundity (200-250\%) (Yue, 1996). To study the nucleic acid sequence of Lrh-1 in $\mathrm{Hu}$ sheep, we cloned and characterized Lrh-1 by rapid amplification of cDNA ends (RACE) followed by bioinformatics. To investigate the distribution of Lrh-1 in Hu sheep, we determined the differential expression of Lrh-1 in some tissues by qRT-PCR. Furthermore, we analyzed the Lrh-1 expression pattern in the hypophysis and hypothalamus during the reproductive cycle. Western blot analysis was performed to analyze changes in the protein level. This study helped expand our knowledge of the biological function of the ovine Lrh-1 gene, explore new methods of reproductive regulation in Hu sheep, and open an avenue of research to unravel the regulatory role of the Lrh-1 gene in tissues.

\section{MATERIAL AND METHODS}

\section{Animals and sampling}

Healthy and multiparous ( 2 to 3 years old) Hu sheep were selected from a stock-seed 
farm in Zhejiang Province, China. The average body weight was $35-40 \mathrm{~kg}$. Before sampling, the animals, in different phases of the estrous cycle, were injected intramuscularly with 0.1 mg cloprostenol sodium (SuZhou Sumu Animal Medical Industry Co., Jiangsu, China). Two days after the injection, all sheep entered estrus. When the sheep entered a 2nd natural estrus, healthy animals were selected at estrus (day 0), metestrus (day 3), diestrus (day 10), and proestrus (day 14) for sampling. Sheep in estrus were determined based on a teaser ram, vaginal examination, ovarian status, and behavior, with a time point assigned as day 0 , and the animal was slaughtered. Next, the metestrus, diestrus, and proestrus animals were slaughtered at days 3,10 , and 14 , respectively. The blood, hypothalamus, hypophysis, oviduct (isthmus), and ovary (non-dominant follicle or non-corpus luteum tissue area) were sampled. Blood samples were centrifuged to collect the serum and then stored at $-20^{\circ} \mathrm{C}$ for reproductive hormone detection. The tissue samples were immediately frozen in liquid nitrogen and stored at $-80^{\circ} \mathrm{C}$ until total RNA and protein extraction.

\section{Sequencing of the Lrh-1 gene from ovine liver}

Total RNA was extracted from tissues using the Trizol reagent (Invitrogen, Carlsbad, $\mathrm{CA}, \mathrm{USA}$ ) according to manufacturer instructions. All RNA samples had an $\mathrm{OD}_{260}: \mathrm{OD}_{280}$ ratio of 1.8-2.0, and the integrity of the total RNA was verified by denaturing agarose gel electrophoresis. Total RNA from tissues was reverse transcribed to obtain 1st-strand cDNA using the PrimeScript RT Reagent Kit (TaKaRa, Dalian, China) and oligo-dT ${ }_{18}$ primers according to manufacturer instructions.

Based on alignment of known Lrh-1 sequences, a set of primers were designed and synthesized. Using the primers (Table 1), a partial cDNA fragment was amplified from the ovine liver. To obtain full-length Lrh-1 cDNA, the RNA-ligase-mediated RACE system (Invitrogen) was used. Primary and nested RACE primers were provided in the kit, and genespecific primers (Table 1) were designed based on a partial fragment of Lrh-1 as per the manufacturer protocol. The RACE amplicons were cloned into the pGEM-T Easy vector, which was then sequenced and compared with known sequences using NCBI-BLAST.

\section{qRT-PCR analysis of Lrh-1}

Total RNA was isolated from various tissues. The absence of genomic DNA contamination in the total RNA was confirmed by non-reverse-transcribed samples as templates. In addition, absence of genomic DNA in the total RNA was ensured by treatment with DNaseI $(\mathrm{TaKaRa})$ before proceeding with 1st-strand cDNA synthesis.

Primers for GAPDH (GenBank accession No. NM_001190390.1), used as the reference gene, and Lrh-1 (Table 1), were designed based on cDNA sequences. All HPLC-grade oligonucleotides were synthesized by Invitrogen. qRT-PCR was performed using an ABI 7500 Real-Time PCR System (Applied Biosystems) with SYBR Green fluorescent labels. Samples (final volume, $25 \mu \mathrm{L}$ ) were run in triplicate and contained the following components: $0.5 \mu \mathrm{L}$ SYBR Green (Invitrogen), $2.5 \mu \mathrm{L} 10 \mathrm{X}$ buffer, $2 \mu \mathrm{L} \mathrm{MgCl}_{2}, 2 \mu \mathrm{L} 10 \mathrm{mM}$ of each dNTP, $0.3 \mu \mathrm{L}$ $50 \mathrm{pM} / \mu \mathrm{L}$ of each primer, $0.3 \mu \mathrm{L}$ Taq (TaKaRa), $15.1 \mu \mathrm{L} \mathrm{ddH}_{2} \mathrm{O}$, and $2 \mu \mathrm{L}$ cDNA template. Amplification was conducted using the following protocol: initial denaturation phase at $95^{\circ} \mathrm{C}$ for $2 \mathrm{~min}, 40$ cycles of $95^{\circ} \mathrm{C}$ for $10 \mathrm{~s}$ for denaturation, then an additional $10 \mathrm{~s}$ for annealing 
(GAPDH: $61^{\circ} \mathrm{C}$; Lrh-1: $58^{\circ} \mathrm{C}$ ), and a final extension step at $72^{\circ} \mathrm{C}$ for $40 \mathrm{~s}$. Melting curve analyses were performed after the RT-PCRs to monitor PCR product purity. Cycle threshold (CT) values were recorded during the exponential phase of the PCR, the expression of Lrh-1 was normalized to that of GAPDH $(\triangle \mathrm{CT}=\mathrm{Lrh}-1 \mathrm{CT}-\mathrm{GAPDH} C \mathrm{CT})$, and the abundance of Lrh-1 mRNA was calculated using the following formula: $2^{-\Delta \Delta C T}$.

\section{SDS-PAGE and Western blot analysis of Lrh-1}

Sheep tissues were homogenized in protein extraction buffer $(10 \mathrm{mM}$ Tris-Cl, $\mathrm{pH}$ 7.4, $0.1 \%$ Triton X-100, $1 \mathrm{mM}$ PMSF, $1 \mathrm{mM}$ EDTA, and $1 \mathrm{mM}$ DTT), followed by centrifugation at $500 \mathrm{~g}$ for $15 \mathrm{~min}$ to remove debris. The supernatant was then subjected to Tris-glycine SDSPAGE with $12 \%$ acrylamide:N,N'-bisacrylamide (30:1). Briefly, the sample was prepared by mixing $100 \mathrm{mg}$ protein homogenate with sample buffer containing $0.125 \mathrm{M}$ Tris- $\mathrm{HCl}, \mathrm{pH} 6.8$, $4 \%$ SDS, $20 \%$ glycerol, $10 \%$ 2-mercaptoethanol, and $0.002 \%$ bromophenol blue, followed by incubation at $95^{\circ} \mathrm{C}$ for $10 \mathrm{~min}$. Tris-glycine ( $25 \mathrm{mM}$ Tris, $192 \mathrm{mM}$ glycine, $\mathrm{pH} 8.3$ ) with $0.1 \%$ SDS was used as the electrode buffer, and the electrophoresis was performed at $120 \mathrm{~V}$. The separated polypeptides were transferred (electroblotted) to nitrocellulose membranes using the Trans-Blot apparatus (Bio-Rad, Hercules, CA, USA).

For immunostaining, the protein blot was treated with $3 \%$ BSA (w/v) in TBS $(10 \mathrm{mM}$

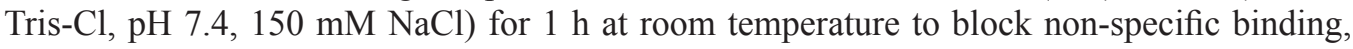
followed by washing with TBST [TBS containing Tween-20 at $0.1 \%(\mathrm{v} / \mathrm{v})]$. The blot was then incubated with Lrh-1-targeting antibodies [Millipore, Billerica, MA, USA; 1:200 dilution in TBST containing 3\% BSA (w/v)] for $2 \mathrm{~h}$ to overnight. After thorough washing with TBST, the blot was incubated with horseradish peroxidase-conjugated secondary antibodies (Santa Cruz Biotechnology, Inc., CA, USA) for $1 \mathrm{~h}$, washed again with TBST, and then colored with DAB. The membrane was scanned using a TANON GIS-2008 gel imager and analyzed by the Tianneng GIS gel image processing system.

\section{Hormone assays}

Sera were collected from the samples. The concentrations of follicle-stimulating hormone (FSH) and luteinizing hormone (LH) were measured in the sera using commercially available radioimmunoassay kits according to manufacturer instructions (Beijing North Institute of Biological Technology, Beijing, China). The assay sensitivity for LH was $1.0 \mathrm{mIU} / \mathrm{mL}$. The intra- and interassay coefficients of variation were 5.1 and $10.6 \%$, respectively. The sensitivity for FSH was $\leq 1.0$ $\mathrm{mIU} / \mathrm{mL}$. The intra- and interassay coefficients of variation were 5.76 and $7.34 \%$, respectively.

\section{Statistical analysis}

The qRT-PCR results are presented as the gene expression of the target gene (Lrh-1) relative to that of the housekeeping gene (GAPDH), and the values for the target gene (Lrh-1) relative to the value of reticulum were determined by the following expression: $2^{-\Delta \Delta C \mathrm{CT}}$. All data were analyzed by one-way ANOVA with the least significant difference post hoc test. Correlations between the qRT-PCR and ELISA data were analyzed by the two-tailed Pearson's correlation. Statistical analyses were performed using the SPSS 13.0 statistical software package. 
$\mathrm{P}<0.05$ was considered to be statistically significant.

\section{RESULTS}

\section{Cloning of Lrh-1 from the liver of Hu sheep}

Full-length Lrh-1 cDNA (1488 bp) was obtained using degenerate primer PCR and 5'- and 3'-RACE (Table 1). The sequence homology of the fragment was determined by NCBIBLAST. The open reading frame encodes a 495 -amino acid residue protein of $56 \mathrm{kDa}$. The nucleotide sequence of Lrh-1 was deposited in GenBank under accession No. JN662490. Twenty different amino acids were detected in Lrh-1. Eight amino acids, Leu (11.11\%), Ser (9.09\%), Lys (6.26\%), Ala (6.06\%), Gln (7.07\%), Glu (6.06\%), Val (5.66\%), and Pro (5.25\%), were predominant. These 8 residues accounted for $56.56 \%$ of all amino acids. ClustalW multiplesequence alignment revealed the presence of DNA-binding and ligand-binding domains that are conserved in transcription factors of the nuclear receptor superfamily (Figure 1).

\begin{tabular}{llc}
\multicolumn{2}{c}{ Table 1. Primer sequences for Lrh-1 RACE and quantitative real-time PCR. } & \\
\hline Primer name & Primer sequence (5'-3') & Function \\
\hline 5'GSP1 & AGGTAGGTGATGTTCCGAGAGCGT & 5'-RACE \\
5'GSP2 & CGAACTTATTCCTCCTCC & 3'-RACE \\
3'GSP1 & CTGTAAGGGCCGACCGAATG & Nested RACE primers \\
'GSP2 & ACCAGACGCTGTTCTCCATCG & \\
P1R & CTGTAAGGGCCGACCGAATG & \\
P2F & AAGCAGTGGTATCAACGCAGAGT & qRT-PCR \\
P2R & CTGTAAGGGCCGACCGAATG & \\
Lrh-1F & GACATCTAAACTAAAGAGCACCA & \\
Lrh-1R & ATCATGGCCTACTTGCAGCA & \\
GAPDH-F & TGTTGCCCAGTAACCAGGAA & \\
GAPDH-R & TCCTGCACCACCAACTGCTT & \\
\hline
\end{tabular}

\section{Expression of Lrh-1 in the hypothalamic-pituitary-gonadal axis and oviduct tissues in ewes}

Lrh-1 mRNA and protein were detected in the hypophysis, hypothalamus, ovary, and oviduct tissues by qRT-PCR and Western blot. A 67-kDa protein was detected on the Western blot, and the distributions of the Lrh-1 protein in tissues correlated well with the transcript levels when probed with the Lrh-1 antibody. The Lrh-1 gene expression in the hypothalamus was at the highest level (Figure 2).

\section{Expression of Lrh-1 in the hypothalamus and hypophysis and the reproductive hormone concentration in serum during the estrous cycle}

The Lrh-1 expression was analyzed in the hypothalamus and hypophysis tissues using qRT-PCR and Western blot during different phases of the estrous cycle of ewes (Figure 3). The expression of Lrh-1 in tissues correlated significantly with mRNA expression, as analyzed by two-tailed Pearson's correlation. The expression of Lrh-1 in the hypophysis was highest 


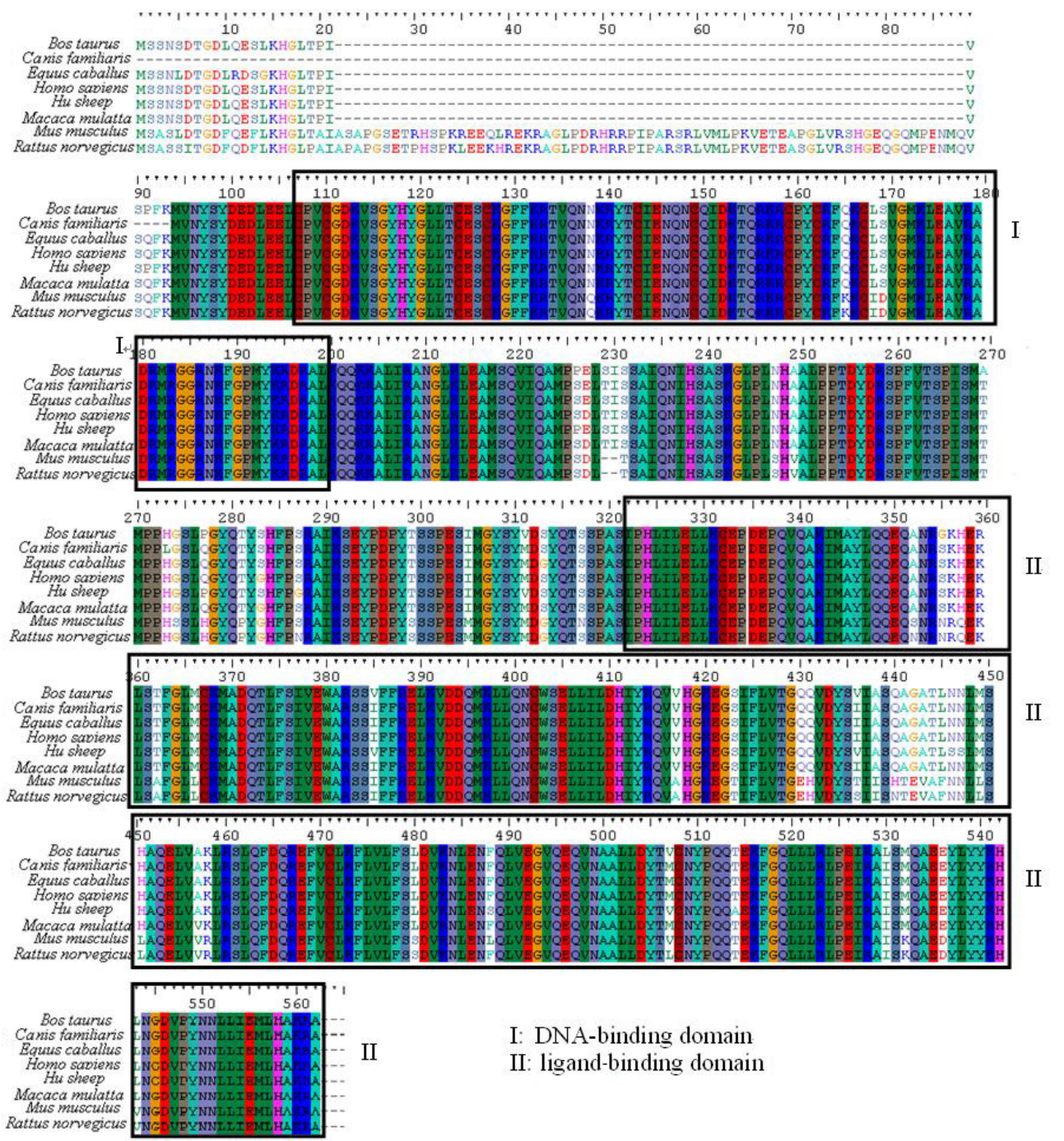

Figure 1. ClustalW alignment of deduced amino acid sequence of Lrh-1 of Hu sheep with other vertebrate counter parts. The alignment was done using the ClustalW software (BioEdit tools). Shaded regions represent conserved amino acids and signature domains are represented by rectangle boxes. GenBank accession numbers of the sequences used are as follows: Bos taurus, DAA21027.1; Equus caballus, NP 001075264.1; Canis familiaris, XP547371.3; Homo sapiens, NP003813.1; Macaca mulatta, XP001110202.1; Mus musculus, NP109601.1; and Rattus norvegicus, Np068510.1.

during estrus and was lowest during the diestrus phase. Lrh-1 expression in the hypothalamus was significant higher during the metestrus phase and lower during the other phases (Figure 3). The FSH and LH levels in the serum of the Hu sheep samples were determined by the RIA method (Table 2). 

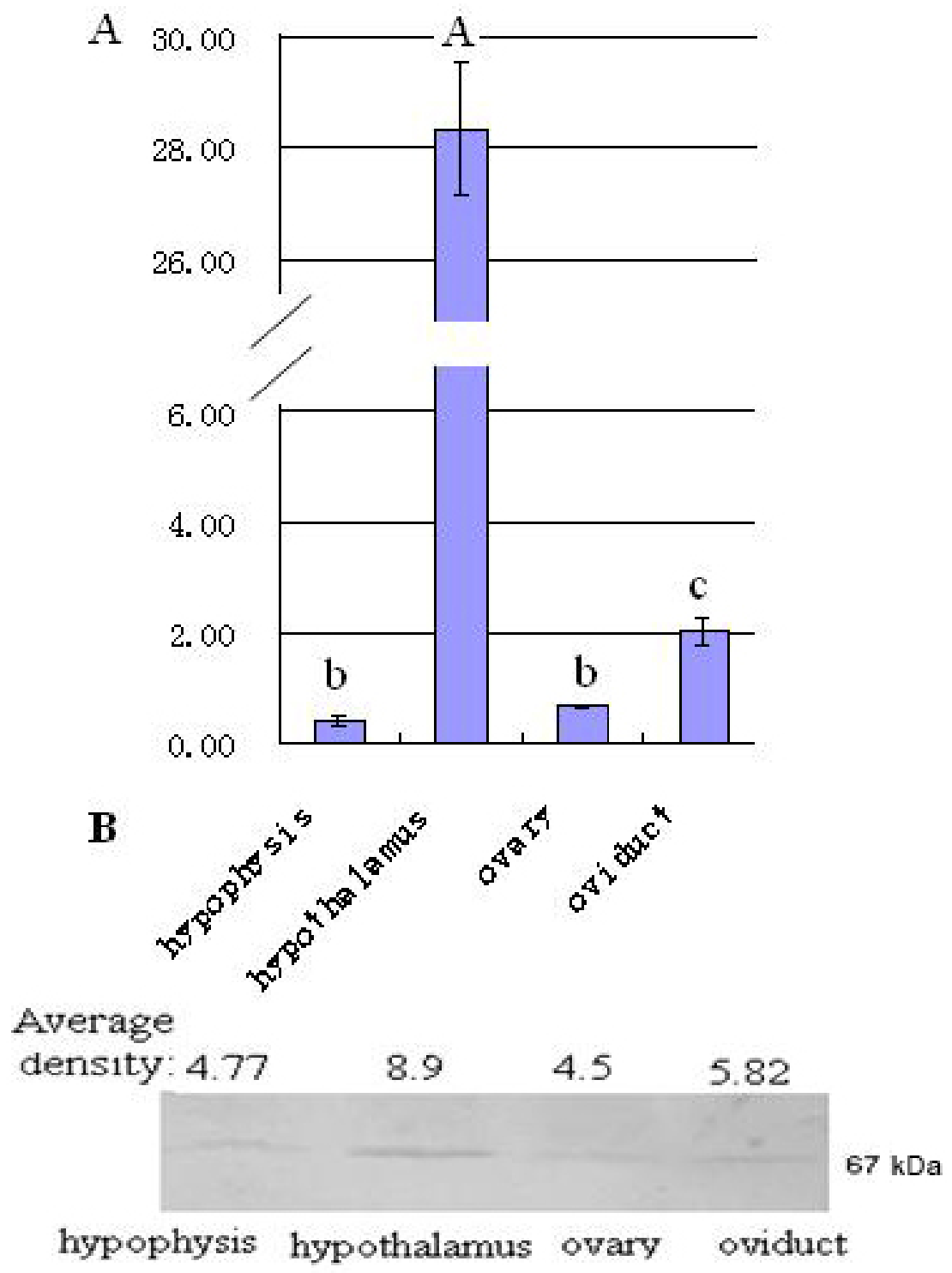

Figure 2. Distribution of Lrh-1 in hypothalamic-pituitary-gonadal axis and oviduct tissues in Hu sheep. A. qRTPCR analysis of Lrh-1 expression was done in tissues (hypophysis, hypothalamus, ovary, and oviduct) of Hu sheep during the metestrus phase. Lrh-1 mRNA expression is normalized to endogenous control GAPDH, and the values for the target gene (Lrh-1) relative to the value of reticulum were determined by the expression: $2^{-\Delta \Delta C T}$. The relative expression level of Lrh-1 mRNA in variant tissues is shown as the mean of three measurements and analyzed by one-way ANOVA. $\mathrm{P}<0.01$ is denoted by the capital letter above the bar. $\mathrm{P}<0.05$ is denoted with different lower case letters above each bar. Groups marked with the same letters are not statistically different. B. Western blot analysis of Lrh-1 expression in tissues (hypophysis, hypothalamus, ovary, and oviduct) of Hu sheep. A 67-kDa protein of Lrh-1 was detected from the result of Western blot. The distributions of the Lrh-1 protein in these tissues were significantly correlated $(\mathrm{P}<0.05)$ with those of the transcript levels when analyzed by two-tailed Pearson's correlation. Pearson's correlation and significant of two-tail value were 0.972 and 0.028 , respectively. 


\section{A.}
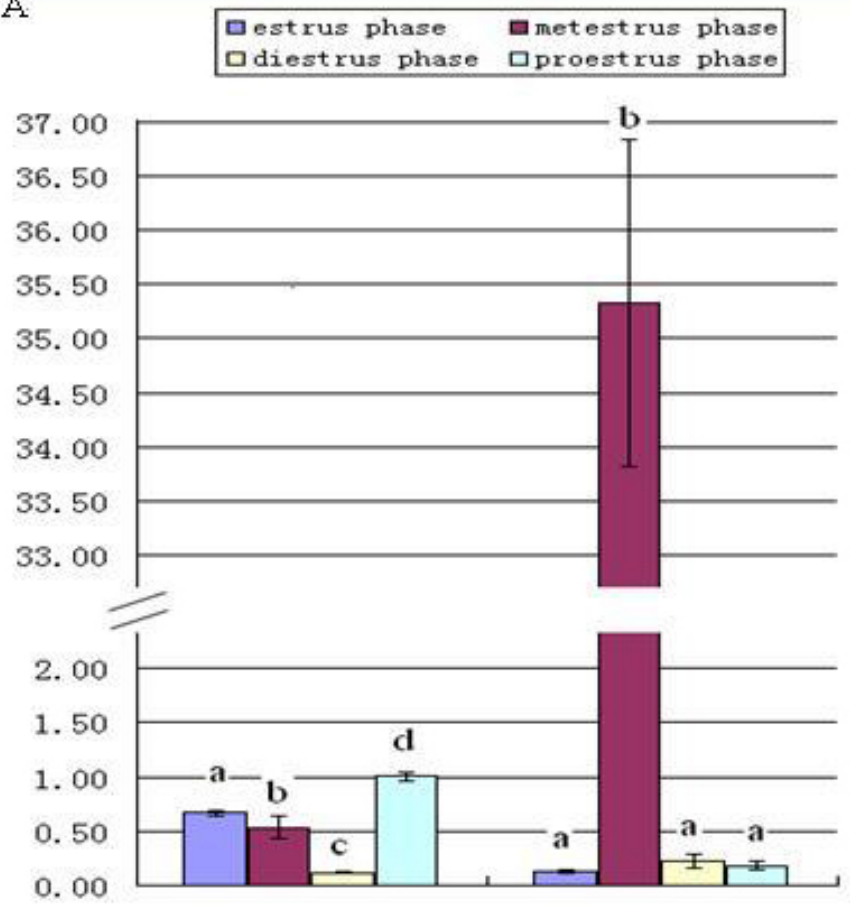

hypophysis

hypothal amus

B
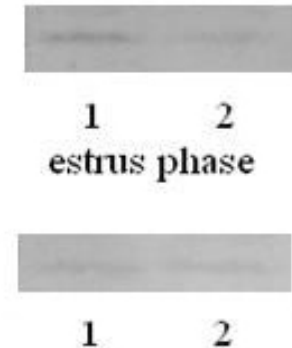

proestrus phase

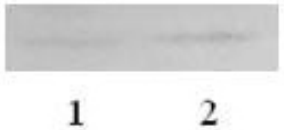

diestrus phase

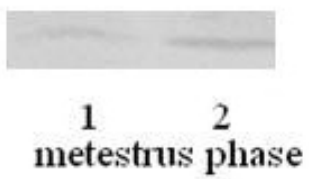

Note: 1 : hypophysis tissue; 2 hypothalamus tissue

Figure 3. Expression of Lrh-1 in hypothalamus and hypophysis of Hu sheep. A. Expression of Lrh-1 in hypothalamus and hypophysis tissues of $\mathrm{Hu}$ sheep during 4 phases of the estrous cycle by qRT-PCR. Quantitative analysis of Lrh1 expression relative to GAPDH expression during different phases of the estrous cycle was reported as relative to the value of hypophysis in proestrus phase calculated using $2^{-\Delta \Delta C T}$ method. Means with different letters differ significantly in one kind of tissues $(\mathrm{P}<0.05)$. B. Lrh-1 protein expressions of hypothalamus and hypophysis tissues were analyzed using Western blot method during different phases of reproductive (ovarian) cycle of female $\mathrm{Hu}$ sheep. Expressions of the Lrh-1 protein in tissues were also significantly correlated with those of the mRNA expression levels analyzed by two-tailed Pearson's correlation $(\mathrm{P}<0.01)$. Pearson's correlation and significant of two-tail value were 0.926 and 0.001 , respectively. 
Table 2. Concentrations of reproductive hormones in Hu sheep samples.

\begin{tabular}{lcc}
\hline Phases & \multicolumn{2}{c}{ Reproductive hormones' concentration } \\
\cline { 2 - 3 } & FSH (mIU/mL) & LH (mIU/mL) \\
\hline Estrus & 1.75 & 3.63 \\
Metestrus & 1.69 & 2.64 \\
Diestrus & 1.51 & 3.12 \\
Proestrus & 1.98 & 2.65 \\
\hline
\end{tabular}

The correlation of Lrh-1 mRNA expression levels and reproductive hormones in hypophysis of Hu sheep among the 4 phases of the estrous cycle was analyzed by two-tailed Pearson's correlation of the SPSS 13.0 software. The results showed that the expressions of Lrh-1 mRNA in hypophysis were significantly correlated with folliclestimulating hormone (FSH) concentration with 0.990 for Pearson's correlation value, while there were no significant correlation with luteinizing hormone (LH) concentration.

\section{DISCUSSION}

To the best of our knowledge, since the discovery of Lrh-1 in mouse liver (GenBank accession No. M81385), this is the first study to analyze the sequence of the Lrh-1 gene in ovine. ClustalW alignment of the deduced amino acid sequence of the cloned ovine Lrh-1 with its vertebrate homologs indicated the presence of typical signature domains, including the DNA-binding and ligand-binding domains of members of the nuclear receptor family, including NR5A (Wang et al., 1989; Honda et al., 1993).

Consistent with previously reported Lrh-1 mRNA expression data (Becker-Andre et al., 1993; Galarneau et al., 1996; Li et al., 1998; Wang et al., 2001; Grgurevic et al., 2005; Higashiyama et al., 2007), Lrh-1 mRNA expression is observed not only in the hypothalamus, hypophysis, ovary, and oviduct tissues but also in the digestive system as well as heart, kidney, spleen, adrenal, lung, and skeletal muscle tissues, as shown in our previous research on $\mathrm{Hu}$ sheep (Wang et al., 2012). This indicated that Lrh-1 plays an essential role in all tissues, and the differential expression may be a function of the innate structures and functions of these tissues. The Lrh-1 protein expression levels in the hypothalamus, hypophysis, ovary, and oviduct tissues were significantly correlated to the mRNA levels in the tissues listed above $(\mathrm{P}<0.05)$.

Previous research on Lrh-1 in ovary tissues demonstrated that Lrh-1 transcripts changed during the estrus phase. Lrh-1 was highly expressed in granulose cells in the ovary during all stages (primary to preovulatory) of follicular development. However, Lrh-1 mRNA expression in the corpus luteum was different: during estrus, luteinized follicles or newly formed corpus luteum exhibited a strong homogeneous labeling pattern, whereas a subset of the corpus luteum from previous cycles exhibited a pattern of weak labeling that was sometimes undetectable, depending on the age of the corpus luteum. The expression of Lrh-1 mRNA within the new corpus lutea declined during metestrus, although some weak signals remained within some corpus lutea from previous cycles. This pattern of labeling was lower or non-discernible in both new and old corpus lutea during diestrus and proestrus (Liu et al., 2003; Higashiyama et al., 2007; Mendelson and Kamat, 2007). However, experiments on Lrh-1 expression in the hypothalamus and hypophysis during the estrous cycle have not been reported. Thus, to our knowledge, this is the first report in ovine to show Lrh-1 expression in the hypothalamus and hypophysis during the estrous cycle. In this study, Lrh-1 mRNA and protein were detected in the hypothalamus and hypophysis of Hu sheep with significant correlation between mRNA expression levels and protein concentrations. 
The hypothalamus synthesizes and secretes neurohormones, e.g., GnRH, which stimulates or inhibits the secretion of pituitary hormones such as LH and FSH. This process controls follicular development and the estrous cycle. The estrous cycle is divided into 4 phases: proestrus, estrus, metestrus, and diestrus, triggered by gonadotropic hormones. A previous report demonstrated that Lrh-1 localizes to the arcuate nucleus of the hypothalamus of mice, based on weak immunostaining (Higashiyama et al., 2007). In the study, Lrh-1 mRNA and protein were detected in the hypothalamus of Hu sheep during the estrous cycle. The Lrh-1 expression levels in the hypothalamus of $\mathrm{Hu}$ sheep were detected at a relatively lower level during all estrous cycles (estrus, diestrus, and proestrus), except during the metestrus phase. An explanation for the significantly higher expression level of Lrh-1 during the metestrus phase requires further research. Lrh-1 gene expression in the hypophysis was significantly different during the 4 estrous cycles $(\mathrm{P}<0.05)$, and the Lrh-1 expression level was the highest during the estrus phase, after which the expression level declined until the diestrus phase; however, during the proestrus phase, the expression level increased. The Lrh-1 gene expression trend was similar to follicular formation in the ovary, which was under the influence of gonadotropic hormones. One or several follicles of the ovary start to grow during the proestrus phase, until the estrus phase, when ovarian follicles are maturing and ovulation occurs. During metestrus, the corpus luteum starts to form, until the diestrus phase when the corpus luteum is active and produces progesterone. To investigate the correlation between Lrh-1 expression levels and reproductive hormone secretion in the hypophysis, we determined the FSH and LH concentration in blood. The results showed that the Lrh-1 expression levels correlated with the FSH concentration $(\mathrm{P}<0.01)$. Therefore, our data, together with a previous report (Higashiyama et al., 2007), suggest that Lrh-1 regulates the gonadotropic hormone secretion activity of the hypophysis, thereby controlling follicular development.

\section{CONCLUSION}

In summary, we cloned Lrh-1 from the liver of Hu sheep. Based on the deduced amino acid sequence homology and structural features, Lrh-1 is a potential nuclear receptor of the NR5A subfamily. Lrh-1 mRNA expression correlated significantly with protein expression in the hypothalamus, hypophysis, ovary, and oviduct tissues. The expression pattern of Lrh-1 in the hypophysis during the estrous cycle was consistent with the reproductive cycle and with FSH synthesis and secretion. In contrast, the Lrh-1 gene expression pattern in the hypothalamus did not change significantly during the estrous cycle, except for the higher expression level observed during the metestrus phase.

\section{ACKNOWLEDGMENTS}

Research supported by the China Agriculture Research System (\#CARS-39) and the Natural Science Foundation of Jiangsu Province, China (\#BK2010356).

\section{REFERENCES}

Becker-Andre M, Andre E and DeLamarter JF (1993). Identification of nuclear receptor mRNAs by RT-PCR amplification of conserved zinc-finger motif sequences. Biochem. Biophys. Res. Commun. 194: 1371-1379.

Clyne CD, Speed CJ, Zhou J and Simpson ER (2002). Liver receptor homologue-1 (LRH-1) regulates expression of 
aromatase in preadipocytes. J. Biol. Chem. 277: 20591-20597.

Delerive P, Galardi CM, Bisi JE, Nicodeme E, et al. (2004). Identification of liver receptor homolog-1 as a novel regulator of apolipoprotein AI gene transcription. Mol. Endocrinol. 18: 2378-2387.

Fayard E, Auwerx J and Schoonjans K (2004). LRH-1: an orphan nuclear receptor involved in development, metabolism and steroidogenesis. Trends Cell Biol. 14: 250-260.

Galarneau L, Pare JF, Allard D, Hamel D, et al. (1996). The alpha1-fetoprotein locus is activated by a nuclear receptor of the Drosophila FTZ-F1 family. Mol. Cell Biol. 16: 3853-3865.

Goodwin B, Jones SA, Price RR, Watson MA, et al. (2000). A regulatory cascade of the nuclear receptors FXR, SHP-1, and LRH-1 represses bile acid biosynthesis. Mol. Cell 6: 517-526.

Grgurevic N, Tobet S and Majdic G (2005). Widespread expression of liver receptor homolog 1 in mouse brain. Neuro. Endocrinol. Lett. 26: 541-547.

Higashiyama H, Kinoshita M and Asano S (2007). Expression profiling of liver receptor homologue 1 (LRH-1) in mouse tissues using tissue microarray. J. Mol. Histol. 38: 45-52.

Honda S, Morohashi K, Nomura M, Takeya H, et al. (1993). Ad4BP regulating steroidogenic P-450 gene is a member of steroid hormone receptor superfamily. J. Biol. Chem. 268: 7494-7502.

Iwaki M, Matsuda M, Maeda N, Funahashi T, et al. (2003). Induction of adiponectin, a fat-derived antidiabetic and antiatherogenic factor, by nuclear receptors. Diabetes 52: 1655-1663.

Li M, Xie YH, Kong YY, Wu X, et al. (1998). Cloning and characterization of a novel human hepatocyte transcription factor, hB1F, which binds and activates enhancer II of hepatitis B virus. J. Biol. Chem. 273: 29022-29031.

Liu DL, Liu WZ, Li QL, Wang HM, et al. (2003). Expression and functional analysis of liver receptor homologue 1 as a potential steroidogenic factor in rat ovary. Biol. Reprod. 69: 508-517.

Lu TT, Makishima M, Repa JJ, Schoonjans K, et al. (2000). Molecular basis for feedback regulation of bile acid synthesis by nuclear receptors. Mol. Cell 6: 507-515.

Luo Y, Liang CP and Tall AR (2001). The orphan nuclear receptor LRH-1 potentiates the sterol-mediated induction of the human CETP gene by liver X receptor. J. Biol. Chem. 276: 24767-24773.

Mendelson CR and Kamat A (2007). Mechanisms in the regulation of aromatase in developing ovary and placenta. $J$. Steroid Biochem. Mol. Biol. 106: 62-70.

Nitta M, Ku S, Brown C, Okamoto AY, et al. (1999). CPF: an orphan nuclear receptor that regulates liver-specific expression of the human cholesterol 7alpha-hydroxylase gene. Proc. Natl. Acad. Sci. U. S. A. 96: 6660-6665.

Pare JF, Roy S, Galarneau L and Belanger L (2001). The mouse fetoprotein transcription factor (FTF) gene promoter is regulated by three GATA elements with tandem E box and Nkx motifs, and FTF in turn activates the Hnf3beta, Hnf4alpha, and Hnflalpha gene promoters. J. Biol. Chem. 276: 13136-13144.

Schoonjans K, Annicotte JS, Huby T, Botrugno OA, et al. (2002). Liver receptor homolog 1 controls the expression of the scavenger receptor class B type I. EMBO Rep. 3: 1181-1187.

Sridevi P, Dutta-Gupta A and Senthilkumaran B (2011). Molecular cloning and expression analysis of fushi tarazu factor 1 in the brain of air-breathing catfish, Clarias gariepinus. PLoS One 6: e28867.

Wang LH, Tsai SY, Cook RG, Beattie WG, et al. (1989). COUP transcription factor is a member of the steroid receptor superfamily. Nature 340: 163-166.

Wang LH, Gao QX, Zhang W and Wang F (2012). Lrh-1 cDNA sequence analysis and tissue expression in Hu sheep. Acta Vet. Zootechnica Sin. 43: 1360-1368.

Wang ZN, Bassett M and Rainey WE (2001). Liver receptor homologue-1 is expressed in the adrenal and can regulate transcription of 11 beta-hydroxylase. J. Mol. Endocrinol. 27: 255-258.

Yue GH (1996). Reproductive characteristics of Chinese Hu sheep. Anim. Reprod. Sci. 44: 223-230. 